Z. Klin. Chem. Klin. Biochem.

10. Jg. 1972, S. $351-354$

\title{
Das Phänomen ansteigender B/F-Kurven bei radioimmunchemischen Bestimmungsmethoden
}

\author{
Von H. J. Bartels, R. D. Hesch und M. Hüfner \\ Technische Assistenz: S. Strohm und W. Becker \\ Medizinische Universitätsklinik Göttingen, Arbeitsgruppe Endokrinologie
}

(Eingegangen am 2. Mai 1972)

In der vorliegenden Arbeit wird das kürzlich bei der radioimmunchemischen Bestimmung von ACTH beobachtete überraschende Bindungsphänomen aufsteigender B/F-Standardkurven durch ein bivalentes Verhalten des Antikörpers vernünftig erklärt. Ein ähnliches Phänomen ist von uns bei Trijodthyronin $\left(T_{3}\right)$-Antikörpern und später auch bei Calcitonin- und Parathormon-Antikörpern festgestellt worden. Dies hat uns zu genaueren Untersuchungen veranlaßt. Dabei wurde ein Zusammenhang zwischen dem an sich für die meisten Antikörper schon bekannten bivalenten Verhalten und aufsteigenden B/F-Standardkurven gefunden. Die erhaltenen theoretischen Resultate haben möglicherweise auch für die radioimmunchemische Bestimmung anderer Hormone praktische Bedeutung: Bei der Messung im aufsteigenden Teil der B/F-Standardkurven kann mit einer Vergrößerung der Empfindlichkeit gerechnet werden.

\section{The phenomenon of the increasing slope of $B \mid F$ curves in radioimmunochemical determination methods}

In the present work it is shown that the recently observed, unexpected ascending of $B / F$ standard curves for the immunochemical determination of ACTH is due to the bivalent behaviour of the antibody. We observed similar behaviour for the antibody binding of triiodothyronine $\left(\mathrm{T}_{3}\right)$ and later of calcitonin and parathormone; the phenomenon was therefore investigated further. Most antibodies are already known to show bivalent properties and this was shown to be correlated with the ascending of the $B / F$ standard curves. The theoretical results obtained are possibly of practical significance for the radioimmunochemical determination of other hormones: sensitivity may be increased by making measurements on the ascending part of the $B / F$ standard curve.

Radioimmunchemische Messungen von Hormonen gehen von folgender Gesetzmäßigkeit aus: Bei der kompetitiven Bindungsreaktion von markiertem und unmarkiertem Hormon mit dem Antikörper wird bei ansteigender Menge unmarkierten Hormons die Bindung des markierten Hormons an den Antikörper verringert. Als Resultat erhält man hyperbelartig abfallende B/F-Standardkurven $(1,2,3)$.

Matsukura und Mitarbeiter (4) beschrieben einen ACTH-Radioimmunoassay mit einem neuen Bindungsphänomen. Die Versuchsergebnisse liefern B/F-Standardkurven, die zunächst ansteigen und nach dem Erreichen eines Maximums den üblichen hyperbelähnlichen Abfall zeigen. Die Höhe des maximalen B/FWertes steigt mit der Konzentration des zugesetzten Antikörpers; der Antigengehalt, bei dem die Standardkurve ihr Maximum erreicht, wächst mit zunehmender Antikörperkonzentration. Das beschriebene, scheinbar paradoxe Bindungsphänomen aufsteigender B/F-Kurven konnte unseres Wissens bislang nicht befriedigend erklärt werden $(4,5)$. Die beobachteten Standardkurven widersprechen offenbar den theoretisch berechneten B/F-Kurven von BERSON und YALOw $(1,6)$.

Bei der radioimmunologischen Bestimmung von menschlichem Parathormon sind ebenfalls aufsteigende B/F-Kurven beobachtet worden, dieser störende Effekt ist hier unter dem Namen "Spur-Effekt" bekannt (7). Bei drei verschiedenen Antisera gegen Trijodthyronin $\left(T_{3}\right)$ fanden wir ein ähnliches, überraschendes Bindungs- verhalten des Antikörpers. Dabei interessierte uns die Frage nach optimalen Versuchsbedingungen für den $\mathrm{T}_{3}$-Radioimmunoassay, $d . h$. die Frage, welche Menge markierten Hormons und welche Antikörperkonzentration ein Optimum an Empfindlichkeit und Präzision (3) ergeben.

Geht man bei der Hormon-Antikörperreaktion von einem Gleichgewicht

$$
\mathrm{H}+\mathrm{AK} \rightleftharpoons \mathrm{HAK}
$$

aus, so gilt nach dem Massenwirkungsgesetz

$$
\mathrm{K}[\mathrm{h}][\mathrm{ak}]=[\mathrm{hak}] \text {. }
$$

Die Affinitätskonstante $\mathrm{K}$ ist für die Frage nach der optimalen Empfindlichkeit bzw. Präzision von erheblichem Interesse $(1,3), \mathrm{K}$ läßt sich mit einfachen Bindungsversuchen (konstante Antikörperkonzentration, variable Menge markierten Hormons, kein unmarkiertes Hormon) bestimmen.

\section{Material und Methodik \\ Antisera}

Uber die Herstellung spezifischer Antikörper gegen $T_{3}$ haben wir kürzlich berichtet ( 8 ).

Die erhaltenen Antisera werden in Endverdünnungen von $1: 10000$ bis $1: 150000$ benutzt.

Die Inkubationszcit für dic Hormon-Antikörpcrreaktion beträgt etwa $24 \mathrm{~h}$.

\section{Lossungen}

Die verwendeten Reagenzien, die Puffer sowic der komplette Inkubationsansatz werden andernorts ausführlich dargestellt (9). 


\section{Arbeitsgang}

Ansteigende ${ }^{125} \mathrm{~J}-\mathrm{T}_{3}$-Konzentrationen werden mit einer konstanten Menge Antikörper inkubiert. Die einzelnen Pipettiervorgänge des vorgenommenen Bindungsversuchs können der Reihenfolge nach in Tabelle 1 abgelesen werden.

Tab. 1

Exemplarisches Ansatzschema für einen Bindungsversuch. Das Gesamtinkubationsvolumen beträgt $0,5 \mathrm{ml}$

\begin{tabular}{ccccc}
\hline $\begin{array}{c}\text { Proben- } \\
\text { nummer }\end{array}$ & $\begin{array}{c}\text { Assay- } \\
\text { Puffer } \\
{[\mu 1]}\end{array}$ & $\begin{array}{c}\text { Antikörper } \\
{[\mu \mathrm{\mu l}]}\end{array}$ & $\begin{array}{c}\text { Normales } \\
\text { Kaninchen- } \\
\text { serum } \\
{[\mu \mathrm{l}]}\end{array}$ & $\begin{array}{c}128 \mathrm{~J}-\mathrm{T}_{3} \\
{[\mu \mathrm{l}]}\end{array}$ \\
\hline $1-2$ & 445 & 50 & - & 5 \\
$3-4$ & 445 & - & 50 & 5 \\
$5-6$ & 440 & 50 & - & 10 \\
$7-8$ & 440 & - & 50 & 10 \\
$9-10$ & 430 & 50 & - & 20 \\
$11-12$ & 430 & - & 50 & 20 \\
$13-14$ & 410 & 50 & - & 40 \\
$15-16$ & 410 & - & 50 & 40 \\
$17-18$ & 390 & 50 & - & 60 \\
$19-20$ & 390 & - & 50 & 60 \\
$21-22$ & 370 & 50 & - & 80 \\
$23-24$ & 370 & - & 50 & 80 \\
$25-26$ & 350 & 50 & - & 100 \\
$27-28$ & 350 & - & 50 & 100 \\
\hline
\end{tabular}

\section{Auswertungsverfabren}

Bei der Berechnung des Verhältnisses B/F von gebundenem $z u$ freiem ${ }^{125} \mathrm{~J}-\mathrm{T}_{3}$-Hormon wird die unspezifische, $\mathrm{d}$. h. die nicht durch den Antikörper bewirkte Bindung folgendermaßen berücksichtigt: Es wird an Stelle des Antiserums normales Kaninchenserum in den Assay eingesetzt (Tab. 1).

Bezeichnet $\mathrm{F}$ die Zählrate (Imp./Min.) des freien ${ }^{125} \mathrm{~J}-\mathrm{T}_{3}$ bei $\mathrm{Zu}$ satz von Antiserum, $T$ die entsprechende Zählrate bei Zusatz von normalem Kaninchenserum, so ist

$$
B / F=\frac{T-F}{F-N} .
$$

Dabei ist $\mathrm{N}$ die Nullrate des Zählgerätes.

Bei der Tabellierung der theoretisch erhaltenen B/F-Standardkurven wurde ein elementares Programm-für die Olivctti Programma 101 verwendet.

\section{Ergebnisse}

Die Ergebnisse des oben beschriebenen Bindungsversuches sind in Abbildung 1, 2 und 3 graphisch wiedergegeben.

In der Terminologie von Berson und Yalow besagt Gleichung (2):

$$
\mathrm{K} \cdot \mathrm{F}([\mathrm{AK}]-\mathrm{B})=\mathrm{B},
$$

dabei bezeichnet [AK] die vor Beginn der Reaktion im Versùchssystem vorhandene Antikörperkonzentration. Gleichung (3) läßt erwarten, daß 1/F als Funktion von $1 / \mathrm{B}$ linear ist und ermöglicht die experimentelle $\mathrm{Be}$ stimmung der gesuchten Konstanten $\mathrm{K}$. Die exhaltenen Resultate (Abb. 1, 2) legen - zumindest für kleine Mengen ${ }^{125} \mathrm{~J}-\mathrm{T}_{3}$ - an Stelle der Gleichungen (1), (2) und (3) eine andere formelmäßige Beschreibung unseres Versuchssystems nahe, nämlich:

und

$$
2 \mathrm{H}+\mathrm{AK} \rightleftharpoons \mathrm{H}_{2} \mathrm{AK}
$$

$$
\mathrm{K}[\mathrm{h}]^{2} \cdot[\mathrm{ak}]=\left[\mathrm{h}_{2} \mathrm{ak}\right]
$$

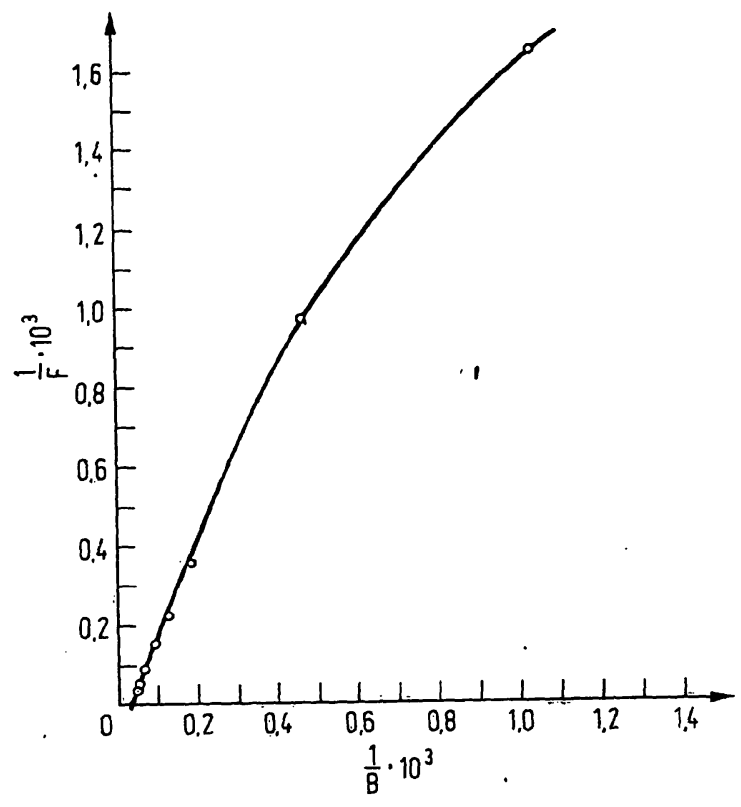

Abb. 1

$1 / \mathrm{F}$ als Funktion von $1 / \mathrm{B}$ bei einem ${ }^{225} \mathrm{~J}-\mathrm{T}_{3}-\mathrm{Bindungsversuch}$. Einge$1 / \mathrm{F}$ als Funktion von 1/B bei eing setzte ${ }^{1.25} \mathrm{~J}-\mathrm{T}_{3}$ Konzentrationen: $4 \mathrm{pg}, 8 \mathrm{pg}$
$80 \mathrm{pg}, 100 \mathrm{pg}$

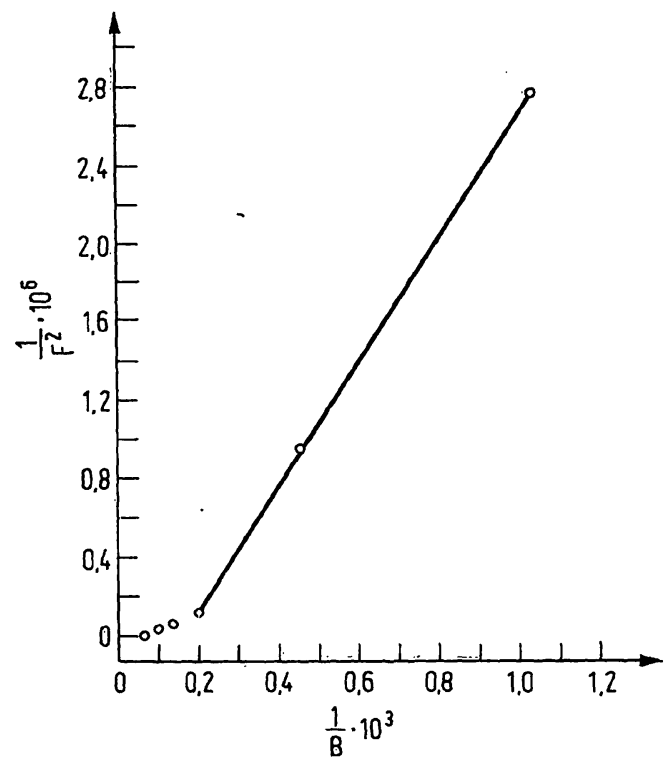

Abb. 2

$1 / \mathrm{F}^{2}$ als Funktion von $1 / \mathrm{B}$ bei demselben Bindungsversuch

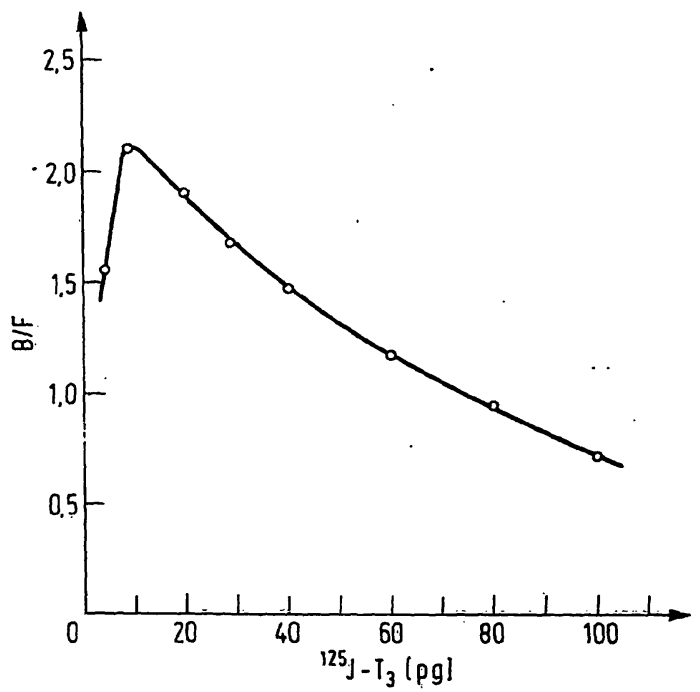

Abb. 3

$B / F$ als Funktion des zugesetzten markierten Trijodthyronins bei demselben Bindungsversuch wie in den Abbildungen 1 und 2 


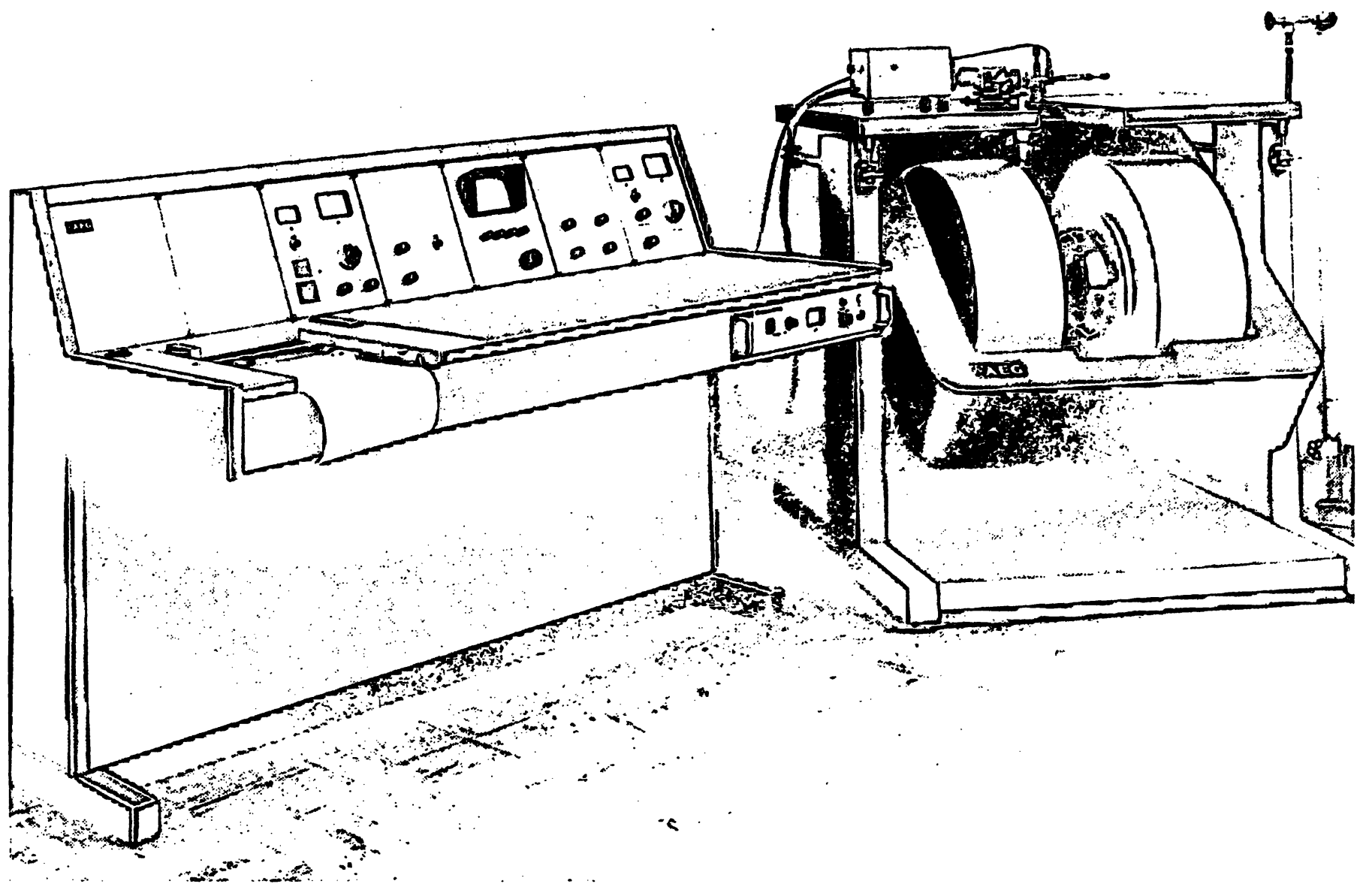

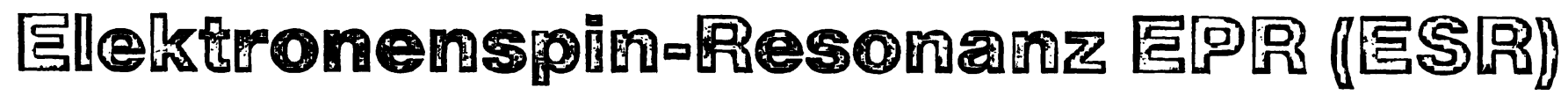

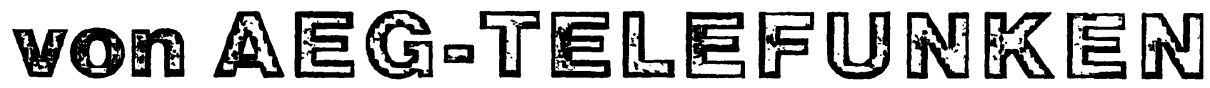

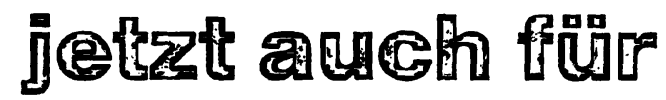 med}

Seit mehr als einem Jahrzehnt ist AEG-TELEFUNKEN auf dem Gebiet der Elektronenspin-Resonanz EPR (ESR) erfolgreich tätig.

Dabei verfolgen wir intensiv die neuen Techniken wie ENDOR und Spinlabeling, die gerade in letzter Zeit auch für die medizinische Forschung aktuell geworden sind.

Unsere Spezialisten und wissenschaftlichen Mitarbeiter beraten Sie gern in allen Einzelheiten.

Wenden Sie sich bitte an
AEG-TELEFUNKEN

Angewandte Elektronik

1 Berlin 65, Drontheimer Str. 35-38

Telefon (03 11) 3912031

\section{AEG}

Elektronenresonanz-

Spektroskopie EPR von AEG-TELEFUNKEN 

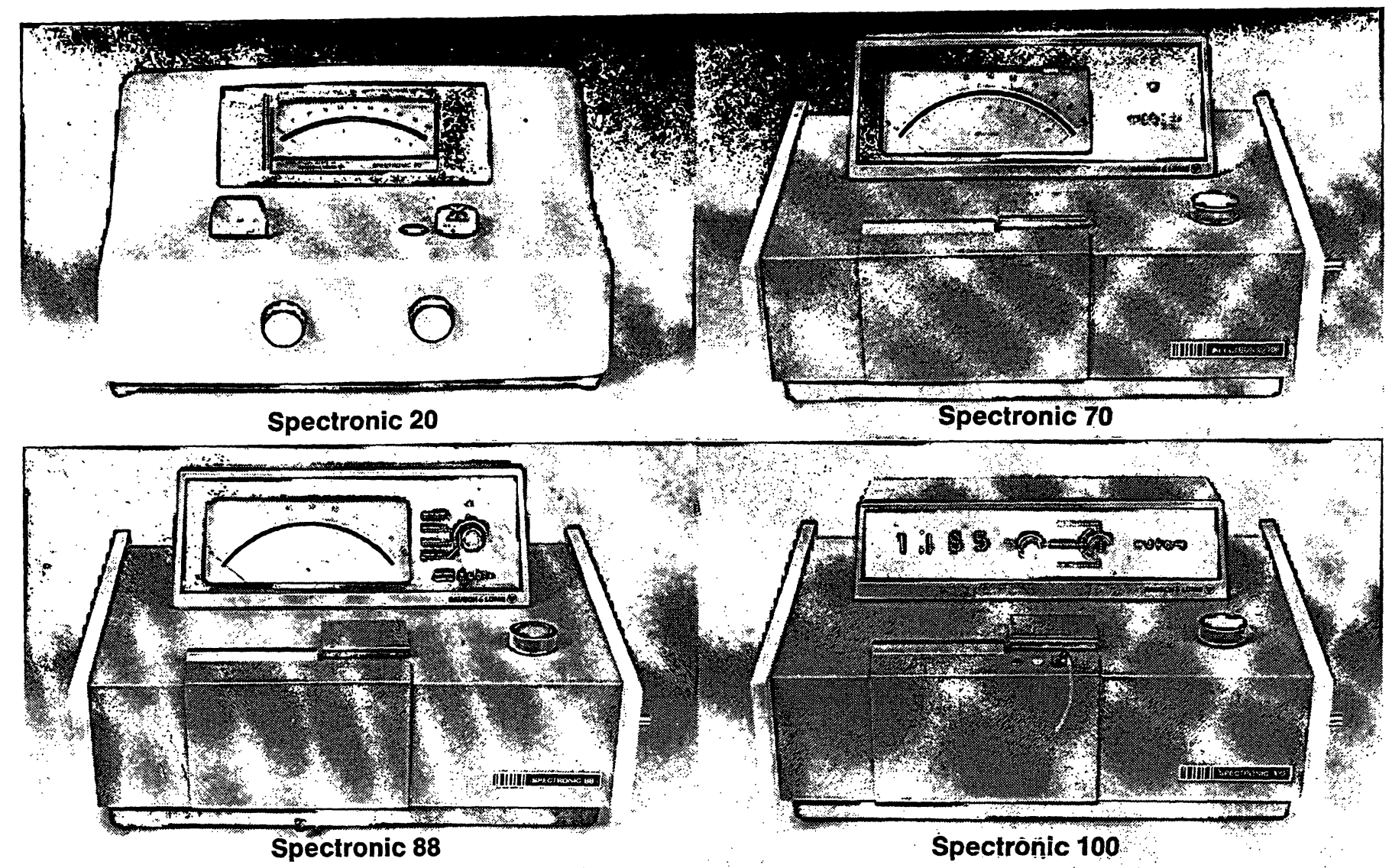

\section{$\mathbb{B A U S C H}$ \& $\mathbb{L O M B}$ hat die Spelktrallphotometer Ihrer Vorstellonng zun Preisen die Sie akzeptieren werden.}

Wie immer auch Ihre

Aufgabenstellung lautet-Bausch \& Lomb bietet Ihnen für jede

Anwendung das optimal konzipierte und preiswerte Gerät.

\section{Spectronic 20}

Gitter-Spektralphotometer mit grosser Anzeigeskala. Wellenlängenbereich $340-950 \mathrm{~nm}$ bei $20 \mathrm{~nm}$ Bandbreite. Das in der Welt meist verwendete Spektralphotometer. Nahezu 100.000 Stück verkauft.

\section{Spectronic 70}

Einfach bedienbares GitterSpektralphotometer. Wellenlängenbereich $325-925 \mathrm{~nm}$ bei $8 \mathrm{~nm}$
Bandbreite. Wenn Sie auf Flexibilität in der Probenraum-Gestaltung Wert legen, so wählen Sie dieses Gerät. Das Gerät ist mit allen StandardKüvetten einschliesslich MikroKüvetten ausrüstbar. Einzel-oder Mehrfach-Probenhalter stehen zur Verfügung.

\section{Spectronic 88}

Das Spectronic 88 erlaubt Direktablesung von Extinktion, Transmission oder Konzentration auf einer $20 \mathrm{~cm}$ langen Spiegelskala. Wellenlängenbereich $325-925 \mathrm{~nm}$.

Extinktionsbereiche 0-1.0 E, 1.0-2.0 E, damit grösstmögliche Ablesegenauigkeit. Zum Gerät ist eine steuerbare Mikro-Durchflussküvette lieferbar.

\section{Spectronic 100}

Digitalanzeigendes Spektralphotometer, linear in E, T oder C. Höchstmöglicher Bedienungskomfort. Überschreitungen des Arbeitsbereiches werden automatisch angezeigt. Zur Messwertverarbeitung stehen Analog- und $B C D$-Ausgänge serienmässig zur Verfügung. Die zum Gerät lieferbare elektronisch gesteuerte MikroDurchflussküvette und die Druckeranschlussmöglichkeit machen das Spectronic 100 zum.Baustein eines automatischen Messplatzes.

\section{Verkauf und Service durch die technischen Büros der Bausch \& Lomb Organisation und den autorisierten Laborfachhandel.}

\section{BAUSCH \& LOMB G G BH ANALYSENSYSTEME}


Dabei bezeichnen [h], $[\mathrm{ak}]$ und $\left[\mathrm{h}_{2} \mathrm{ak}\right]$ jeweils die sich beim Gleichgewicht einstellenden Konzentrationen der betreffenden Substanzen. [H], [AK] seien die zu Anfang vorhandenen Konzentrationen.

Dann ist

$$
\begin{gathered}
B=2\left[\mathrm{~h}_{2} \mathrm{ak}\right] \\
F=[\mathrm{h}]=[\mathrm{H}]-2\left[\mathrm{~h}_{2} \mathrm{ak}\right] \\
B / F=\frac{2\left[\mathrm{~h}_{2} \mathrm{ak}\right]}{[\mathrm{H}]-2\left[\mathrm{~h}_{2} \mathrm{ak}\right]}
\end{gathered}
$$

Also:

und wegen

$$
2\left[\mathrm{~h}_{2} \mathrm{ak}\right]=\frac{[\mathrm{H}] \cdot \mathrm{B} / \mathrm{F}}{1+\mathrm{B} / \mathrm{F}},
$$

$$
[\mathrm{h}]=[\mathrm{H}]-2\left[\mathrm{~h}_{\mathbf{2}} \mathrm{ak}\right]=\frac{[\mathrm{H}]}{1+\mathrm{B} / \mathrm{F}}
$$

gilt dann nach Gleichung (5):

$$
\mathrm{K}:\left(\frac{[\mathrm{H}]}{1+\mathrm{B} / \mathrm{F}}\right)^{2}\left([\mathrm{AK}]-1 / 2 \cdot \frac{[\mathrm{H}] \cdot \mathrm{B} / \mathrm{F}}{1+\mathrm{B} / \mathrm{F}}\right)=1 / 2 \frac{[\mathrm{H}] \cdot \mathrm{B} / \mathrm{F}}{1+\mathrm{B} / \mathrm{F}}
$$

Mit den Abkürzungen

gilt also:

$$
R=B / F, x=[H], C=[A K]
$$

oder

$$
K \cdot x\left(2 C-\frac{x R}{1+R}\right)=R(1+R)
$$

$$
2 \mathrm{CKx}(1+\mathrm{R})-\mathrm{Kx}^{2} \mathrm{R}=\mathrm{R}(1+\mathrm{R})^{2}
$$

Es interessiert $B / F$ als Funktion von $[\mathrm{H}]$. Durch die Gleichung (7) bzw. (6) wird $R=B / F$ als Funktion von $\mathrm{x}=[\mathrm{H}]$ implizit beschrieben. Um die für eine explizite Beschreibung von $R$ notwendige Auflösung einer Gleichung dritten Grades - etwa mit den CARDANoschen Auflösungsformeln - zu vermeiden, kann man (7) lokal nach $\mathbf{x}$ auflösen, was weniger Mühe bereitet:

Nach Gleichung (6) folgt:

$$
\begin{gathered}
x^{2}-\frac{2 C(1+R)}{R} x+\frac{(1+R)^{2}}{K}=0 \\
x=C \cdot \frac{1+R}{R} \pm \sqrt{C^{2} \cdot \frac{(1+R)^{2}}{R^{2}}-\frac{(1+R)^{2}}{K}}(G \mid .8)
\end{gathered}
$$

Bestimmt man die Konstanten $\mathrm{C}=[\mathrm{AK}]$ und $\mathrm{K}$ nach Gleichung (5), so liefert Gleichung (8) den in Abbildung 4 wiedergegebenen, von einem Computer tabellierten Kurvenverlauf. Dieser stimmt mit den experimentellen Ergebnissen überein, die erhaltenen B/F-Kurven haben genau die in 1. c. (4) als paradox angesehene Gestalt.

$\mathrm{Da}$ man sich für möglichst sensitive Radioimmunoassays interessiert, liegt die Frage nach der Bestimmung der Lage des maximalen $\mathrm{B} / \mathrm{F}$-Wertes, insbesondere die Frage nach der Abhängigkeit von der gewählten Antikörperkonzentration nahe. Es wird eine mathematische Bedingung für den maximalen B/F-Wert erhalten, indem man die erste Ableitung in diesem Punkt gleich Null setzt.

Anwendung des Operators $\frac{d}{d x}$ auf Gleichung (7) liefert zusammen mit der Extremwertbedingung

$$
\begin{gathered}
\frac{d R}{d x}=\frac{d B / F}{d[H]}=0 \\
2 C K(1+R)-2 \mathrm{KxR}=0
\end{gathered}
$$

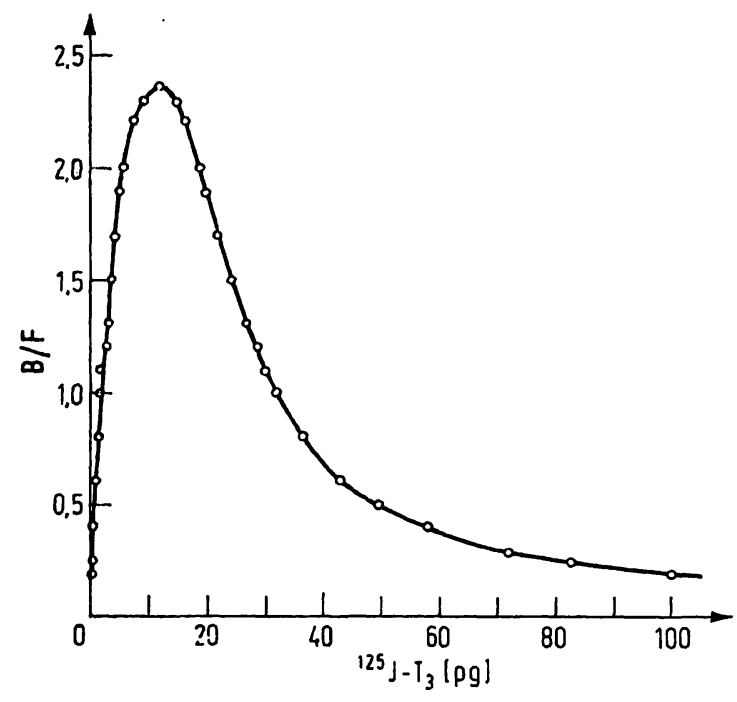

Abb. 4

Die nach Formel (8) von einem Computer tabellierte B/F-Kurve

d. h.

$$
\mathrm{x}=\frac{1+\mathrm{R}}{\mathrm{R}} \cdot \mathrm{C}
$$

Wegen Gleichung (8) gilt diese Gleichung dann und nur dann, wenn der Radikand in Gleichung (8) Null ist, d. h. wenn

$$
C^{2} \cdot \frac{(1+R)^{2}}{R^{2}}=\frac{(1+R)^{2}}{K}
$$

also

gilt.

$$
K C^{2}=R^{2}
$$

Damit hat man dann

$$
(B / F)_{\max }=\sqrt{K} \cdot[A K]
$$

Für den zugehörigen $[\mathrm{H}]-$ Wert hat man dann nach Gleichung (8):

$$
[\mathrm{H}]_{\max }=[\mathrm{AK}] \frac{1+\sqrt{\mathrm{K}}[\mathrm{AK}]}{\sqrt{\mathrm{K}} \cdot[\mathrm{AK}]}=\frac{1}{\sqrt{\mathrm{K}}}+[\mathrm{AK}] .
$$

Die Gleichungen (9) und (10) beschreiben die Lage des Maximums in Abhängigkeit von der Antikörperkonzentration, diese Ergebnisse stimmen qualitativ mit experimentellen Befunden überein (4).

\section{Diskussion}

Unsere Untersuchungen zeigen, daß das zunächst paradox erscheinende Phänomen aufsteigender B/FKurven auf das an sich für die meisten Antikörper schon bekannte $(10,11,12)$ bi- bzw. multivalente Verhalten dieser Antikörper zurückgeführt werden kann.

Radioimmunologische Hormonbestimmungen zeichnen sich gegenüber anderen Meßmethoden durch eine große Empfindlichkeit und Präzision aus. Bei einigen Radioimmunoassays genügt allerdings auch die dort erreichte Empfindlichkeit noch nicht, um befriedigende Meßwerte angeben zu können: Bei der in unserem Labor praktizierten radioimmunchemischen Bestimmungsmethode für menschliches Calcitonin (HCT) 
wiesen 5 von 14 Normalpersonen HCT-Spiegel unterhalb der Nachweisgrenze auf (13).

Die Frage, inwieweit sich eine Vergrößerung der Sensitivität bzw. Präzision bei radioimmunologisschen Hormonnachweisen erreichen läßt, ist daher beim HCT oder auch beim thyreotropen Hormon (TSH) (1. c. (14), S. 262) von großem klinischen Interesse.

Mit der für das $T_{3}$ geschilderten experimentellen Überprüfung des Antikörperbindungsverhaltens ${ }^{1}$ ) muß festgestellt werden, ob bei ähnlichen Ergebnissen eine Messung von Hormonkonzentrationen im ansteigenden Teil der B/F-Kurven möglich ist und damit eine Vergrößerung der Empfindlichkeit erreicht werden kann: Der in 1. c. (4) angegebene ACTH-Radioimmunoassay kann zwischen 0 und $1 \mathrm{pg}$ ACTH unterscheiden (1. c. (4), S. 495)! Wegen Gleichung (9) und (10) wird man bei derartigen Versuchen mit hohen Antikörperkonzentrationen arbeiten müssen.

Die anfangs gestellte Frage nach den optimalen Versuchsbedingungen, von der wir ausgegangen sind, kann

1) Erste Bindungsversuche haben beim HCT und bei unseren Antiseren einen noch ausgeprägteren ansteigenden Teil der B/FKurven ergeben als beim $\mathrm{T}_{3}$. momentan von uns noch nicht beantwortet werden. Immerhin, das oben geschilderte Phänomen aufsteigender B/F-Kurven zeigt nach unserer Auffassung, $\mathrm{da} B$ Zurückhaltung gegenüber den sich zum Teil widersprechenden bisher gefundenen Ergebnissen zur Charakterisierung optimaler Versuchsbedingungen $(1,3)$ angebracht erscheint. Aufsteigende B/F-Kurven sind unseres Wissens im Zusammenhang mit diesen Fragen bislang nicht diskutiert wordeń; in der Nähe des Maximums dieser B/F-Kurven ist eine Messung von Hormonkonzentrationen notwendig unpräzis. Keine der genannten Theorien berücksichtigt diese Möglichkeit $(1,3)$. Will man wie üblich in dem abfallenden Teil der B/F-Kurven messen, so wird man die zugesetzte Menge markierten Hormons etwas größer als $[\mathrm{H}]_{\max }$ wählen müssen, um einen Verlust an Empfindlichkeit $\mathrm{zu}$ vermeiden.

Bei unserem Modell, bei unserer-formelmäßigen $\mathrm{Be}$ schreibung aufsteigender B/F-Kurven, bleiben unberücksichtigt: die Heterogenität der Antikörper, mögliche Hormon-Antikörperkomplexe wie HAK, $\mathrm{H}_{3} \mathrm{AK}_{2}$ usw. und verschiedenes Verhalten von markiertem und unmarkiertem Hormon gegenüber dem Antikörper.

\section{Literatur}

1. Berson, S. A. und R. S. Yalow, Clin. Chim. Acta, Amsterdam 22, 51 (1968). - 2. EkrNs, R. P., G. B. Newman und J. L. O' RIORDAN, In: Hayes, R. L., F. A. Goswitz und B. E. P. Murphy (eds.), Radioisotopes in Medicine: In Vitro Studies, US Atomic Energy Commission, Oak Ridge (1968) S. 59. - 3. Ekrns, R. P. und G. B. Newman, Acta Endocr., Supplement 147, 11 (1970). 4. Matsukura, S., C. D. West, Y. Ichikawa, W. Jubiz, G. Harada und F. H. Tyler, J. Laborat. Clin. Med., S. Louis 77, 490 (1971). - 5. Odelx, W. D. und W. H. Daughaday, Principles of Competitive Protein-Binding Assays, Lippincott Co., Philadelphia (1971) S. 22. - 6. Berson, S. A. und R. S. YALOw, J. clin. Invest. 38, 1996 (1959). - 7. The Wilson Laboratories, Bull. No. 16, Chicago, July 26 (1971). - 8. Hesch, R. D. und M. HüFNER, Acta biol. med. germ. 5, 28 (1972). - 9. HEsCH, R. D., M. HüFNER und A. v. z. MüHLEN, Germ. Med. 2, 18 (1972). 10. Humhrey, J. H. und R. G. White, Kurzes Lehrbuch der Immunologie, Georg Thieme Verlag, Stuttgart (1971) S. 231. 11. Pauling, L., J. Amer. Chem. Soc. 62, 2643 (1940). - 12. Pauling, L., D. Pressman, D. H. Cambeli und C. Ikeda, J. Amer. Chem. Soc. 64, 3003 (1942). - 13. HürnER, M. und R. D. Hesch, Klin. Wschr. 49, 1149 (1971). - 14. v. z. Mühlen, A. und D. EMrICH, diese Z. 9, 257 (1971).

\section{H.-J. Bartels}

Med. Univ.-Klinik-Arbeitsgruppe Endokrin. 34 Göttingen Humboldtallee 1 